\title{
Diversité végétale et usages des plantes dans une zone de savane soudanienne : Cas de la localité de Ferkessédougou (Nord, Côte d'Ivoire)
}

\author{
Abdoulaye CISSE*, Mevanly OUATTARA, Estelle Anny N'GUESSAN et \\ Joël Emmanuel N'Gouan ABROU
}

Université Félix Houphouët-Boigny, UFR Biosciences, Laboratoire de Botanique, Côte d'Ivoire, 22 BP 582

Abidjan 22, Côte d'Ivoire.

*Auteur correspondant ; E-mail : abdoulayeciss@rocketmail.com

\section{RESUME}

En Côte d'Ivoire, principalement dans le nord, on observe la présence de plusieurs formations végétales très variées allant des forêts claires et aux différents faciès de savanes. Dans la localité de Ferkessédougou, une étude a été menée dans le but de connaître la flore, la végétation et de l'usage des plantes. Cette étude a permis de recenser 192 espèces de végétales. Elles se répartissent en 148 genres et 64 familles. Parmi ces espèces, certaines ont été identifiées comme alimentaires, médicinales ou à divers autres usages. Aussi, vingt sont considérées comme des espèces ayant un statut particulier et qui nécessite une attention particulière en vue de leur protection ainsi que les habitats où elles se rencontrent. La présence de ces espèces peut constituer des indicateurs clés pour le suivi environnemental de la zone d'étude.

(C) 2020 International Formulae Group. All rights reserved.

Mots clés : Diversité végétale, Services écosystémiques, savane soudanienne, Ferkessédougou, Côte d'Ivoire.

\section{Plant diversity and plant uses in a Sudanese savannah area: Case of the locality of Ferkessédougou (North, Côte d'Ivoire)}

\begin{abstract}
In Ivory Coast, mainly in the north, we observe the presence of several very varied plant vegetations anging from open forests to different facies of savannah. In the locality of Ferkessédougou, a study was carried out to learn about the flora, vegetation and the use of plants. This study identified 192 species of plants. They are divided into 148 genera and 64 families. Among these species, some have been identified as food, medicinal or various other uses. Also, twenty are considered to be species with a special status and which requires very special attention for their protection as well as the habitats where they occur. The presence of these species can constitute key indicators for the environmental monitoring of the study area.

(C) 2020 International Formulae Group. All rights reserved.
\end{abstract}

Keywords: Plant diversity, Ecosystem services, Sudanese savannah, Ferkessédougou, Ivory Coast. 


\section{INTRODUCTION}

Les forêts représentent un intérêt socioéconomique pour les populations locales dans le monde et en Afrique (Badjare et al., 2018). Elles sont un bien commun universel et une source de nombreux services pour l'homme (Tankoano, 2017). En effet, ces ressources permettent aux hommes de satisfaire leurs besoins fondamentaux, constituent le socle de la conservation de la biodiversité et conditionnent la présence des espèces végétales et animales (N'Guessan et al., 2019).

En dépit de leur importance, les ressources naturelles en l'occurrence les forêts subissent une forte pression humaine qui constitue aujourd'hui une menace pour la pérennisation des biens et services qu'elles fournissent. La perte de ces ressources provoque un affaiblissement et de la capacité des communautés tributaires des forêts à en tirer des revenus, de la nourriture et des médicaments. Aujourd'hui, le taux de déforestation sur le continent africain ne fait que s'accroître (FAO, 2012).

En Côte d'Ivoire, la couverture forestière est passée de 16 millions à 2,5 millions d'hectares en 2016 (Aké-Assi \& Boni, 1990). Les conséquences de la régression du couvert forestier sur la biodiversité et l'environnement sont entre autres la fragmentation du couvert végétal, le changement climatique et la diminution des services écosystémiques. En effet, la diminution des ressources ligneuses impacte négativement les services écosystémiques tels que les services de prélèvement pour l'alimentation, l'énergie, la construction, la médecine, l'artisanat et autres que procure la biodiversité aux populations locales (Traore et al., 2011 ; Davranche et Taïbi, 2015 ; Ouattara et al., 2016), particulièrement dans la zone soudanienne.

Les formations végétales savanicoles couvrent plus de la moitié de la Côte d'Ivoire. Le bois énergie constitue une source de revenus non négligeable. En effet, des tonnes de bois de feu et de charbon de bois sont acheminées chaque jour des zones rurales vers les villes (N'Klo, 2012). Ainsi, 90\% environ de la population urbaine des zones de savanes utilisent le bois de feu ou le charbon de bois et $100 \%$ des ménages ruraux utilisent le bois de chauffe (Ouattara, 2001).

La culture itinérante sur brûlis et l'élevage extensif, systèmes de culture fortement consommateurs d'espace, sont malheureusement très utilisés dans la région. La création de vastes blocs agro-industriels (complexes sucriers et blocs aménagés du projet soja au Nord-Ouest du pays) ainsi que les divers aménagements agro-pastoraux et les feux de brousse sont autant de facteurs qui entament notablement l'équilibre écologique. Ainsi, par la destruction de leurs habitats, par le surpâturage ou tout simplement suite à une exploitation abusive, certaines espèces forestières sont en voie d'extinction (Fao, 2001).

Face aux pressions anthropiques impactant la pérennisation des espèces végétales dans la vie socio-économique des communautés locales et dans la disponibilité alimentaire et médicinale, plusieurs études mettant en exergue les usages de ces espèces végétales prélevées par les populations locales ont été menées en Côte d'Ivoire (Kouamé et al., 2008 ; Dro et al., 2013 ; Vroh et al, 2014 ; Ouattara et al., 2016 ; Tiébré et al., 2016 ; Yao et al., 2018). Toutefois la plupart de ces études ont été menées dans la partie sud forestière du pays (Aké-Assi, 2002 ; Ouattara et al., 2013), et, très peu d'études se sont intéressées à la diversité floristique savanicole du nord et particulièrement celle de la zone soudanienne de la Côte d'Ivoire (Ambé, 2001).

Cette étude se veut une contribution à la connaissance des plantes utilitaires en zone savanicole au Nord de la Côte d'Ivoire. De façon générale, il aborde la composition floristique, la disponibilité et l'utilisation des plantes de la localité de Ferkessédougou au Nord de la Côte d'Ivoire. Plus spécifiquement, il s'agit d'évaluer la richesse et la diversité floristique du milieu, les fonctions et usages que les plantes revêtent pour les communautés locales. 


\section{MATERIEL ET METHODES Site d'étude}

La présente étude a été réalisée au Nord de la Côte d'Ivoire, dans le District des Savanes, précisément dans la circonscription administrative du Tchologo, ayant pour cheflieu de région, le département de Ferkessédougou.

Cette région regroupe également les départements de Kong et Ouangolodougou. Selon Guillaumet et Adjanohoun (1971), le Nord de la Côte d'Ivoire appartient au domaine soudanais caractérisé par des forêts claires et des savanes (savanes boisées, arborées, arbustives et herbeuses) qui en dérivent. Ce domaine a pour limite méridionale les savanes guinéennes et les forêts denses semi-décidues. $\mathrm{Au}$ nord de cette limite, toute savane intégralement protégée devient une forêt claire typique : au Sud de cette limite, toute savane protégée de la même façon devient une forêt dense de type semi-décidu. Cette limite est une limite climatique qui sépare le groupe climatique II (forêts claires et savanes soudaniennes) et le groupe climatique III (forêts denses semi-décidues et savanes guinéennes) comme l'a mentionné Mangenot (1951).

(Figure 1).

\section{Collecte des données \\ Inventaire floristique et dendrométrique}

Dans la présente étude, deux techniques de relevés de terrain complémentaires ont été utilisées pour la collecte des données. Il s'agit du relevé de surface et du relevé itinérant. Le relevé de surface a consisté à délimiter des parcelles de $100 \mathrm{~m}^{2}(10 \mathrm{~m} \mathrm{x} 10 \mathrm{~m})$ dans les différents biotopes rencontrés, notamment: Cultures annuelles, Forêts claires, Forêts galeries, Forêts sèches, Jachères, Plantation d'anacarde, Plantation de canne à sucre, Savane arborée, Savane arbustive et Savane herbeuse (Ouattara et al., 2014).

Une fois les placettes délimitées, leurs coordonnées géographiques sont enregistrées à l'aide du récepteur GPS. A l'intérieur de ces placettes, la présence de toutes les espèces rencontrées a été notée. En ce qui concerne les espèces arborescentes dont le Diamètre à Hauteur de Poitrine est supérieur à $2,5 \mathrm{~cm}$, le nombre d'individus a été compté et les circonférences ont été également mesurées. Le choix d'un DBH minimum de 2,5 cm permet de maximiser la diversité des espèces végétales. Des relevés itinérants ont été réalisés dans tous les espaces inventoriés. Ce relevé a permis de recenser les espèces non rencontrées dans les relevés de surface afin de compléter la liste floristique générale.

\section{Enquête ethnobotanique}

Dans les villages les plus proches des sites d'inventaire, des interviews auprès de personnes-ressources ont été réalisées pour appréhender l'importance culturelle des espèces végétales. Ces personnes ressources étaient principalement composées de tradithérapeutes, de paysans et de féticheurs. Des questionnaires ont été soumis aux communautés locales afin de collecter des données sur l'utilisation des espèces dans divers domaines traditionnels. Cet aspect du travail est important d'autant plus que le mode d'utilisation des espèces par les populations locales est un facteur de destruction des peuplements sauvages mais aussi un moyen de conservation de certaines espèces (Ouattara et al., 2016). En effet, les différents domaines d'utilisation, les types d'usages et le type d'organe utilisé des espèces ont été renseignés. Au total 30 personnes ont été interviewées dans les villages visités que sont Amaravogo, Tchègbo, Pissankaha, Kotiénédougou et Karnavogo (Figure 1).

\section{Analyse des données}

Pour la gestion et l'analyse des données, les données floristiques ont été saisies dans le logiciel Excel. Avec ce logiciel, des tableaux croisés dynamiques, des histogrammes et des graphiques à secteurs ont été réalisés ainsi que des fréquences des espèces ont été calculées. A l'aide du logiciel MVSP 3.1, des indices de diversité ont été calculés.

La richesse spécifique représente le nombre total ou moyen d'espèces par unité de surface ou le nombre d'espèces que compte une communauté ou un peuplement (Ramade, 2005). Elle a été obtenue par addition du 
nombre total d'espèces par type de biotope. Les genres et les familles des espèces ont été également dénombrés. Pour chaque biotope, le type biologique de toutes les espèces a été déterminé en se référant à plusieurs auteurs (Raunkiaer, 1934 ; Schnell, 1971 ; Keay et Hepper, 1954-1972 ; Aké-Assi, 2001, 2002). La chorologie de toutes les espèces a été également déterminée en utilisant les grandes subdivisions phytogéographiques de White (1983). Elle a permis de distinguer quatre types chorologiques d'espèces en fonction de leur répartition dans les régions phytogéographiques. Ce sont les espèces appartenant à la région Guinéo-Congolaise (GC), Soudano-Zambézienne (SZ), la zone de transition entre ces deux grandes régions (GC$\mathrm{SZ}$ ) et les espèces introduites (i). Les noms des espèces ont été mis à jour à partir de Lebrun et Stork (1991-1997) utilisé par plusieurs botanistes (Aké-Assi, 2001 ; Aké-Assi, 2002). La nomenclature des espèces, suivie dans cette étude, est celle d'APG IV (2016). Dans le cadre de-cette étude, il a été déterminé les espèces à statut particulier en vue de souligner l'importance des différents biotopes en termes de conservation pour la biodiversité. En effet, un accent a été mis sur les espèces (GCW) qui sont des espèces endémiques aux forêts de l'Afrique de l'Ouest, ainsi que les espèces endémiques à la Côte d'Ivoire (GCi) et aux forêts de la région phytogéographique de la Haute Guinée (HG) (Ouattara et al., 2016). Pour la caractérisation de toutes ces espèces endémiques, la liste des espèces inventoriées a été confrontée au catalogue des plantes vasculaires de la Côte d'Ivoire de Aké-Assi $(1984 ; 1998 ; 2001 ; 2002)$ et la liste de Jongkind (2004). Cette dernière liste a été utilisée uniquement pour la détermination des espèces endémiques des forêts de la Haute Guinée (HG). Pour la détermination des espèces rares et/ou menacées d'extinction de la flore ivoirienne, des espèces vulnérables et en danger, il a été confronté également la liste des espèces inventoriées à celle de Aké-Assi (1998 ; 2001 ; 2002) et la liste rouge de l'Union Internationale pour la Conservation de la nature (UICN, 2018).

\section{Diversité floristique}

Pour la caractérisation de la diversité $\alpha$ ou diversité intrazonale, les indices de Shannon-Weaver ( $\left.\mathrm{H}^{\prime}\right)$ et d'équitabilité ou d'équipartition de Piélou (J) ont été calculés pour apprécier la diversité spécifique de chaque biotope. Ces deux indices sont donnés par les formules mathématiques suivantes:

$$
H^{\prime}=-\sum[(n i / N) x \ln (n i / N)]
$$

Dans cette formule, $\mathrm{H}$ ' désigne l'indice Shannon, ni désigne le nombre d'individus d'une espèce $\mathrm{i}$ et $\mathrm{N}$ désigne le nombre total des individus de toutes les espèces.

$$
\mathrm{J}=\mathrm{H}^{\prime} / \ln \mathrm{S}
$$

Dans cette formule, $\mathrm{J}$ désigne l'indice d'équitabilité ou d'équipartition de Piélou, $\mathrm{H}$ ' est l'indice de Shannon, $S$ est le nombre total d'espèces d'un biotope donné, et $\ln (\mathrm{S})$ représente la diversité maximale du biotope. Le Coefficient de similitude de Sørensen a été également calculé pour mesurer le degré de ressemblance floristique entre la flore des différents biotopes. La formule utilisée est celle proposée par Sørensen (1948). Il se calcule de la manière suivante :

$$
\mathrm{Ks}=\frac{2 c}{a+b} \times 100
$$

Dans cette formule, Ks représente le coefficient de similitude de Sørensen ; a est le nombre d'espèces d'une liste appartenant à un site $\mathrm{A} ; \mathrm{b}$ est le nombre d'espèces d'une liste appartenant à un site $\mathrm{B}$ et $\mathrm{c}$ désigne le nombre d'espèces communes aux deux sites (A et $\mathrm{B}$ ) que l'on veut comparer. Ce coefficient varie entre 0 et $100 \%$. L'hypothèse de similitude ou ressemblance est admise, lorsque le coefficient est supérieur à 50\% (Troupin, 1966).

Au niveau ethnobotanique, les enquêtes ont été combinées aux inventaires floristiques et ont porté sur l'utilisation faite de certaines espèces végétales par les populations locales. Concrètement, il s'agissait de parcourir avec 
des riverains, l'ensemble des biotopes afin, d'identifier et renseigner les différentes espèces (noms vernaculaires ou communs) qui ont un intérêt particulier à leurs yeux.

Concernant l'usage des espèces, les informations sur ceux-ci ne sauraient être exhaustives. La présente étude a juste montré l'importance de certaines espèces pour les communautés locales vivant sur le site d'étude et ses environs.

\section{Traitement statistique des données}

Pour réaliser les analyses statistiques, des données floristiques ont été saisies dans le logiciel Excel. Ensuite, le test paramétrique d'ANOVA a été réalisé pour la comparaison des moyennes des valeurs de ces indices après confirmation de la normalité par le test de Shapiro. Chaque fois que la probabilité calculée était significative, le test de Tukey a été effectué pour la comparaison deux à deux des moyennes en vue d'apprécier les différences significatives qui existent entre celles-ci.

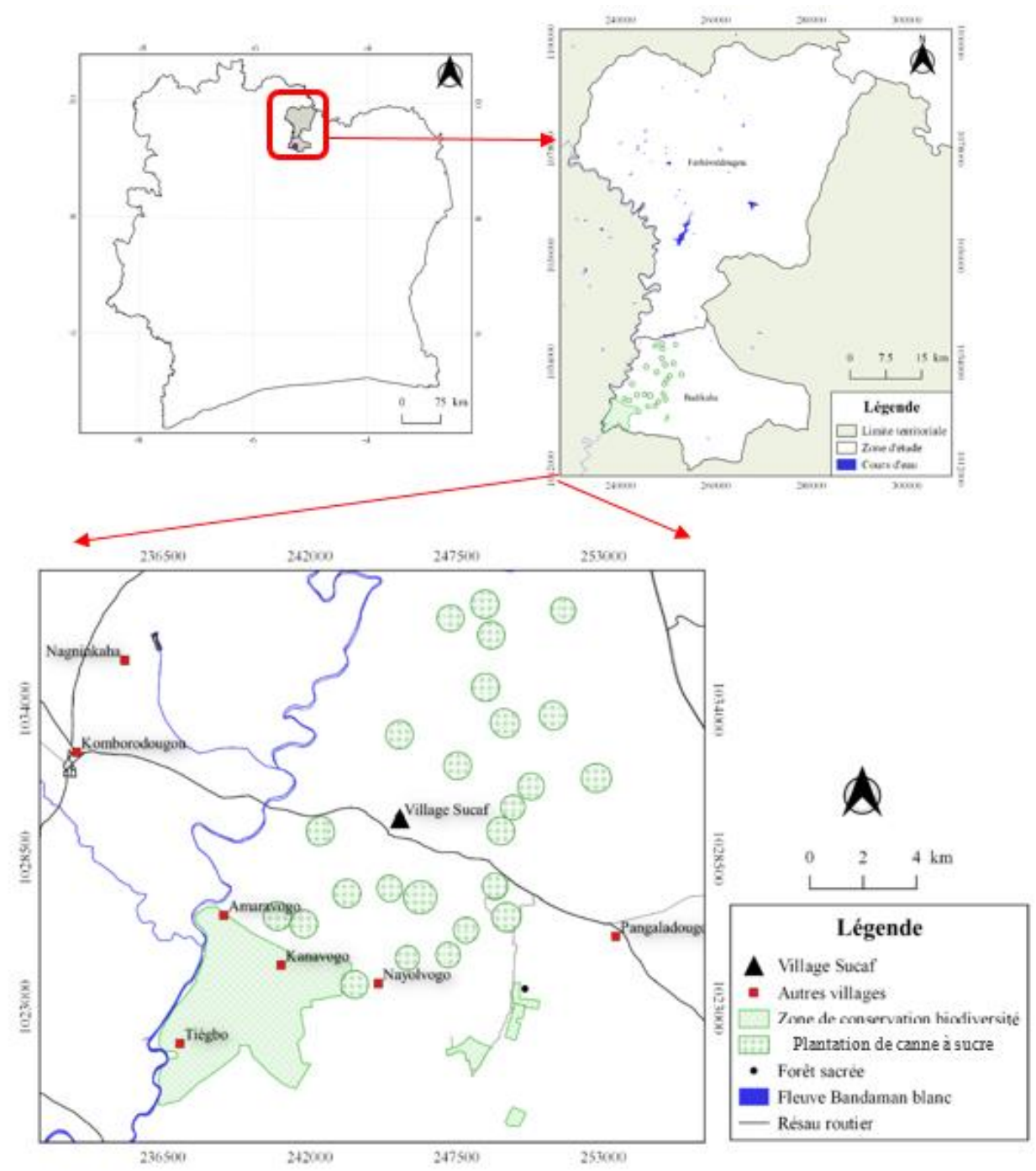

Figure 1 : Localisation de la zone d'étude. 


\section{RESULTATS}

\section{Types de végétation du site d'étude}

Les observations faites sur le terrain ont permis de déterminer les principaux types de formations végétales présents sur le périmètre sujet à l'étude. Il s'agit des formations typiques du domaine soudanien: îlots de forêts sèches, des forêts claires relativement dégradées, des savanes arborées, des savanes arbustives, des galeries forestières ainsi que des formations végétales anthropisées à savoir des jachères de différents âges et des sites de cultures annuelles et/ou pérennes.

\section{Richesse et composition floristiques}

L'étude de la flore a permis de recenser 192 espèces de plantes. Elles se répartissent en 148 genres et 64 familles. Trente-deux (32) genres comprennent au moins deux (2) espèces et 116 genres sont représentés par une seule espèce. Les genres les plus représentés sont : Ficus (7 espèces), Combretum (5 espèces) et Cola (4 espèces). Les familles les plus riches en espèces sont les Fabaceae, les Euphorbiaceae et les Moraceae avec chacune $7 \%$ des espèces inventoriées. Cinquante-sept (57) familles comprennent au moins 1 espèce chacune (Figure 2). Elles renferment, à elles seules, $59 \%$ des espèces recensées.

L'ensemble du site d'étude présente une richesse moyenne qui varie de $10,7 \pm 11,2$ à $89,7 \pm 67,8$ espèces (Tableau 1). Ainsi, l'analyse de la flore de ce site montre que les forêts galerie sont les plus riches avec un nombre de 108 espèces. Tandis que les cultures annuelles sont les moins riches. Elles possèdent au total de 17 espèces. Les différences entre les moyennes de la richesse ont été significatives (Test de Kruskal Wallis : $\chi^{2}=83,3 ; p<$ 0,0001).

Les espèces les plus prépondérantes du site d'étude selon leur fréquence d'apparition dans les différents biotopes-sont les suivantes : Anacardium occidentale (dans les plantations d'anacarde) ; Vitellaria paradoxa, Sida urens, Nauclea latifolia, Lophira lanceolata (dans les cultures annuelles); Flacourtia indica, Anthonotha macrophylla, Mimusops kummel, Pterocarpus santalinoides (dans les forêts galeries) ; Cassia sieberiana, Daniellia oliveri Anogeissus leiocarpus (dans les jachères).

Au total, 12 types biomorphologiques ont été recensés sur le site du projet. Les microphanérophytes (mp) sont les plus nombreux. Ils représentent $59 \%$ des espèces (Figure 3). Parmi ces microphanérophytes, les formes lianescentes sont au nombre de 30 (14\% de l'ensemble des espèces) alors que les formes arborescentes, plus nombreuses sont au nombre de 86 espèces $(45 \%)$.

Les espèces qui se rencontrent naturellement dans la zone de transition forêtsavane (GC-SZ) sont les plus nombreuses. Elles représentent $44,27 \%$ de l'ensemble des espèces (Figure 4). Elles sont suivies par les espèces de la région Guinéo-Congolaise $(30,73 \%)$. Les espèces exotiques introduites (i) sont les moins nombreuses. Elles représentent seulement $3,64 \%$ du cortège floristique.

\section{Qualité de la flore : présence d'espèces à statut particulier \\ Espèces endémiques}

Dans l'ensemble des espèces recensées, cinq (5) sont signalées comme des espèces endémiques (Tableau 2). Il s'agit de Uvaria tortilis A. Chev. Ex Hutch. \& Dalziel, un taxon endémique à la Côte d'Ivoire (GCi). Cette espèce a été recensée principalement dans les forêts claires et les jachères. Puis, quatre taxons endémiques du bloc forestier ouest africain (GCW) ont été identifiés. Ces espèces sont Dalbergia oblongifolia G. Don, Milicia regia A. Chev. (Figure 2), Samanea dinklagei (Harrns) Keay et Cola caricaefolia (G. Don) K. Schum. Ces espèces ont été rencontrées dans des forêts sèches, forêts claires, des jachères et des aires de cultures. Les espèces Aeglopsis 
chevalieri Swingle et Cola caricaefolia (G. Don) K. Schum sont des espèces de la Haute Guinée (HG).

\section{Espèces rares et/ou menacées d'extinction}

Les inventaires ont permis de recenser 18 espèces menacées sur la liste rouge de l'UICN (2018). Parmi celles-ci, l'on distingue sept (07) espèces Vulnérables (VU) dont Vitellaria paradoxa C. F. Gaert; deux (02) espèces Quasi Menacée (NT ou LR/nt) ; deux (02) espèces Peu concernée (LC); et Absence de données (DD) avec Raphia Sudanica. Huit (08) espèces sont considérées comme plantes rares et envoie d'extinction de la flore ivoirienne. Il s'agit entre autres de Milicia excelsa (Welw.) Benth., Syzygium guineense (Willd.) DC. var. guineense, Pericopsis laxiflora (Harms) Meeuwen, Lannea nigritana (Sc. Elliot) Keay var. nigritana.

\section{Indices de diversité}

$\mathrm{Eu}$ égard aux valeurs moyennes de l'indice de diversité de Shannon qui varient de $2,15 \pm 0,07$ bits dans les forêts claires à $1,36 \pm$ 0,17 bits dans les savanes herbeuses, les différents biotopes étudiés sont relativement moins diversifiés (Tableau 3). Il n'existe aucune différence statistique entre ces valeurs moyennes $(F=1,66 ; p=0,14)$. En ce qui concerne l'indice d'équitabilité, il existe une différence significative entre les différentes valeurs moyennes observées dans les biotopes $(F=4,65 ; p<0,05)$. La valeur la moins élevée est obtenue dans les forêts galeries $(0,50 \pm$ $0,05)$ et la plus élevée dans les savanes herbeuses $(0,8 \pm 0,1)$ où il existe une bonne équipartition des espèces (Tableau 3 ).

\section{Ressemblance floristique entre les différents espaces}

Selon le coefficient de similitude de Sørensen, les ressemblances entre les différents biotopes pris deux à deux se situent seulement entre les jachères et les savanes arborées d'une part et entre les jachères et les savanes arbustives d'autre part. Tous les autres biotopes présentent des cortèges floristiques spécifiques différents (Tableau 4).

\section{Domaines d'utilisation : usages locaux et types d'organes utilisés des espèces végétales}

Ce sont au total 30 personnes qui ont été interrogées lors des enquêtes. Elles sont de 06 personnes par village. La plupart des personnes interviewées sont des autochtones senoufos.

Sur l'ensemble des personnes enquêtées, on a enregistré $2,48 \%$ de femmes et $97,52 \%$ d'hommes. La moyenne d'âge des personnes enquêtées est de 38,9 $\pm 10,4$ ans. Le niveau d'instruction des personnes enquêtées varie d'une localité à une autre. Ainsi la proportion des enquêtés non scolarisés est de $54 \%$; ce qui revient à seulement $46 \%$ de personnes lettrées. Dans les localités visitées, les personnes mariées sont été les plus abondantes.

Sur l'ensemble des 192 espèces inventoriées, plusieurs sont prélevées par les populations pour divers usages. Les principaux domaines d'utilisation sont l'alimentation, la médecine traditionnelle, l'artisanat et la construction. Ainsi, 39 espèces sont comestibles (Tableau 5) et les organes les plus consommés sont les fruits, les rhizomes, la tige. On n'enregistre que 31 espèces qui sont utilisées dans le traitement de diverses pathologies (Tableau 6). Vingt-sept (27) espèces servent à d'autres usages dont la construction, le fourrage, l'artisanat et l'ornementation (Tableau 7). 


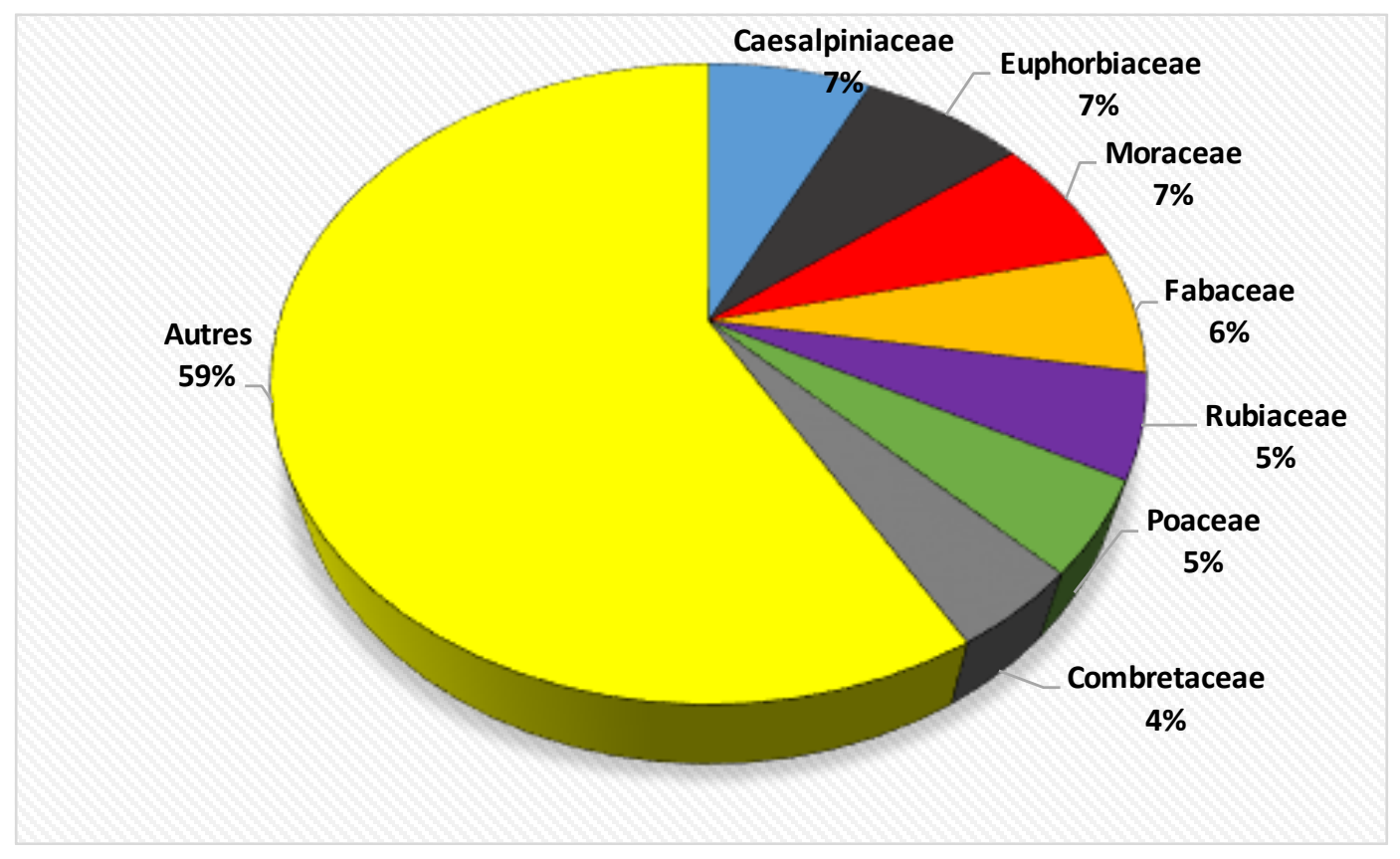

Figure 2 : Proportions des différentes familles du site d'étude.

Tableau 1: Valeurs des paramètres de la richesse floristique.

\begin{tabular}{|c|c|c|c|}
\hline Habitat & Richesse spécifique & Richesse spécifique moyenne & Test statistique \\
\hline Culture annuelle & 17 & $12,8 \pm 9,8^{\mathrm{de}}$ & \multirow{9}{*}{$\begin{array}{c}\chi 2=83,3 ; p \\
0,0001\end{array}$} \\
\hline Forêt claire & 92 & $52,4 \pm 58,7^{\mathrm{ab}}$ & \\
\hline Forêt galerie & 108 & $89,7 \pm 67,8^{\mathrm{a}}$ & \\
\hline Forêt sèche & 81 & $51,4 \pm 61,6^{\mathrm{b}}$ & \\
\hline Jachère & 49 & $22,6 \pm 25,8^{\mathrm{d}}$ & \\
\hline Champ anacardiers & 28 & $18,1 \pm 13,4^{\mathrm{d}}$ & \\
\hline Savane arborée & 64 & $24,7 \pm 28,1^{\mathrm{c}}$ & \\
\hline Savane arbustive & 60 & $24,6 \pm 41,4^{\mathrm{c}}$ & \\
\hline Savane herbeuse & 21 & $10,7 \pm 11,2^{\mathrm{e}}$ & \\
\hline
\end{tabular}




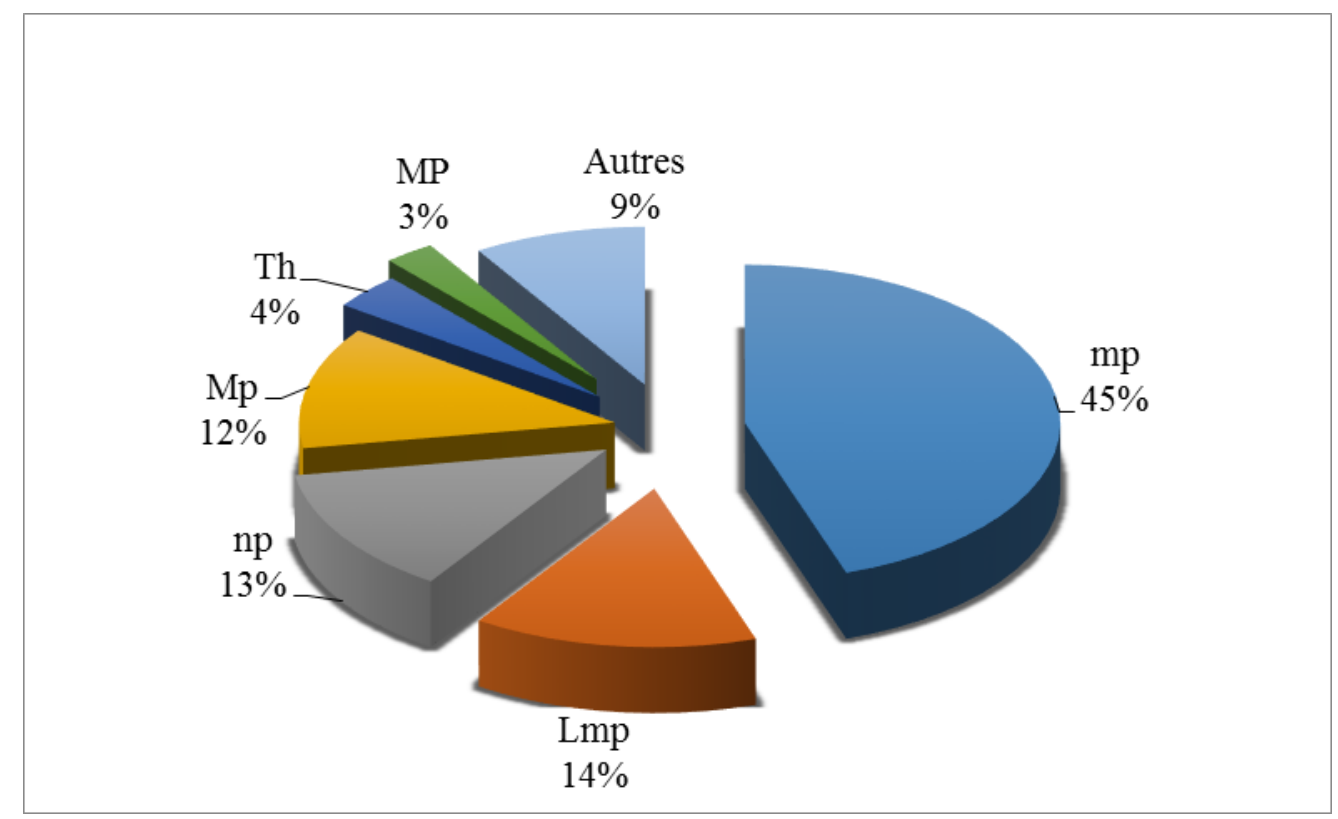

Figure 3: Répartition des types biomorphologiques.

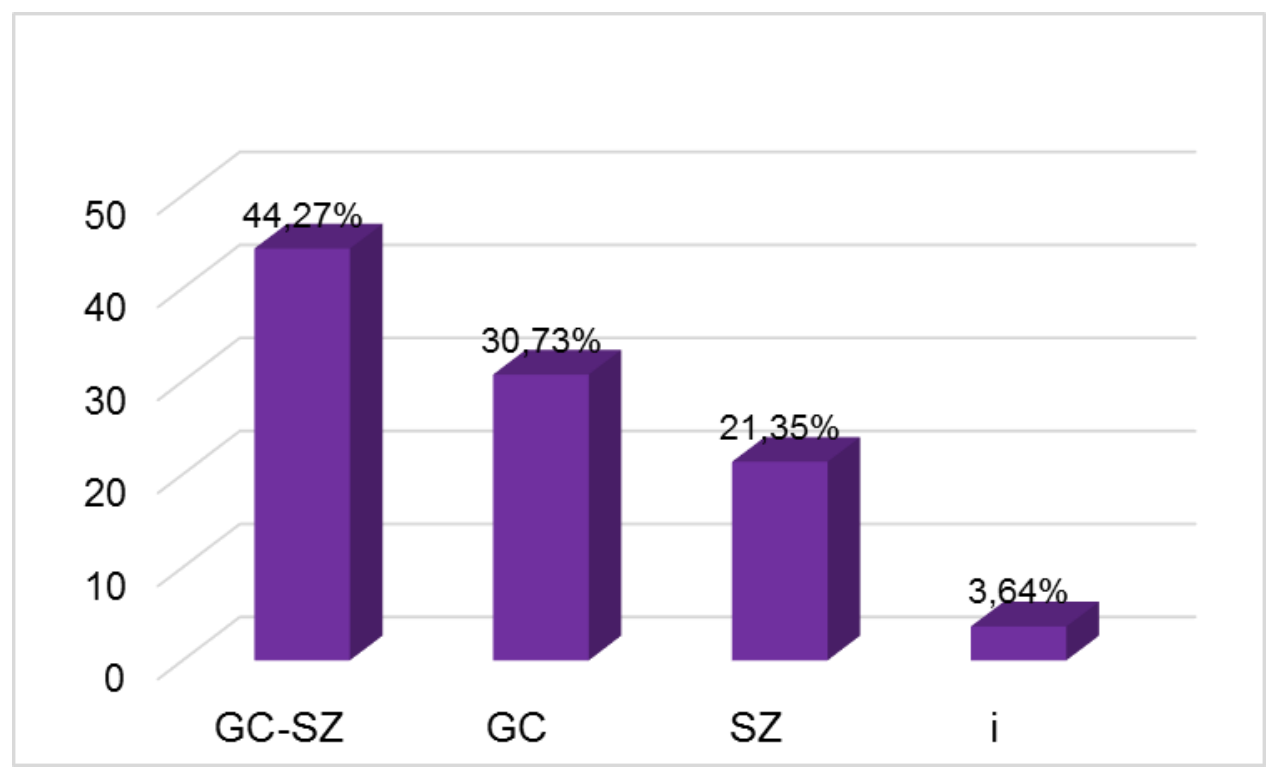

Figure 4 : Repartition phytogéographoque des espèces végétales recensées sur le site du projet. GC : espèces de la région Guineo-Congolaises ; SZ : espèces de la région Soudano-Zambéziennes ; GC-SZ : espèces de la zone de transition forêt-savane ; $\mathrm{i}$ : espèces exotiques introduite. 
Tableau 2 : Liste des espèces endémiques du site d'étude.

\begin{tabular}{llll}
\hline Espèces & Familles & Endémisme & Biotopes \\
\hline Uvaria tortilis & Annonaceae & GCi & Forêt claire dégradée/Jachère à Chromolaena \\
\hline Dalbergia oblongifolia & Fabaceae & GCW & Forêt sèche/Culture \\
\hline Milicia regia. & Moraceae & GCW & Culture/Forêt sèche/Jachère à Chromolaena \\
\hline Samanea dinklagei & Mimosaceae & GCW & Forêt sèche/Forêt claire dégradée \\
\hline Cola caricaefolia & Sterculiaceae & HG_GCW & Forêt sèche \\
\hline Aeglopsis chevalieri & Rutaceae & HG_GC & Forêt sèche \\
\hline
\end{tabular}

Tableau 3 : Indices de diversité spécifique des différents biotopes.

\begin{tabular}{lcc}
\hline Biotopes & Indice de Shannon & Indice d'équitabilité \\
\hline Cultures annuelles & $1,56 \pm 0,33$ & $0,79 \pm 0,07^{\mathrm{b}}$ \\
\hline Forêts claires & $2,15 \pm 0,07$ & $0,63 \pm 0,071^{\mathrm{ab}}$ \\
\hline Forêts galeries & $1,47 \pm 0,08$ & $0,50 \pm 0,05^{\mathrm{a}}$ \\
\hline Forêts sèches & $1,93 \pm 0,44$ & $0,63 \pm 0,02^{\mathrm{ab}}$ \\
\hline Jachères & $1,90 \pm 0,31$ & $0,65 \pm 0,06^{\mathrm{ab}}$ \\
\hline Plantation anacarde & $1,53 \pm 0,33$ & $0,65 \pm 0,071^{\mathrm{ab}}$ \\
\hline Plantation canne villageoise & $1,62 \pm 0,07$ & $0,75 \pm 0,07^{\mathrm{ab}}$ \\
\hline Savane arborée & $1,58 \pm 0,59$ & $0,75 \pm 0,16^{\mathrm{b}}$ \\
\hline Savane arbustive & $1,76 \pm 0,06$ & $0,67 \pm 0,02^{\mathrm{ab}}$ \\
\hline Savane herbeuse & $1,36 \pm 0,17$ & $0,81 \pm 0,13^{\mathrm{b}}$ \\
\hline Statistique du test & $F=1,66 ; p=0,14$ & $F=4,65 ; p<0,05$ \\
\hline
\end{tabular}


Tableau 4: Ressemblance floristique entre les différents biotopes.

\begin{tabular}{|c|c|c|c|c|c|c|c|c|c|c|}
\hline & $\begin{array}{l}\text { Cultures } \\
\text { annuelles }\end{array}$ & $\begin{array}{l}\text { Forêt } \\
\text { claire }\end{array}$ & $\begin{array}{l}\text { Forêt } \\
\text { galerie }\end{array}$ & $\begin{array}{l}\text { Forêt } \\
\text { sèche }\end{array}$ & Jachère & $\begin{array}{l}\text { plantation } \\
\text { anacarde }\end{array}$ & $\begin{array}{l}\text { Plantation Canne } \\
\text { villageoise }\end{array}$ & $\begin{array}{l}\text { Savane } \\
\text { arborée }\end{array}$ & $\begin{array}{l}\text { Savane } \\
\text { arbustive } 1\end{array}$ & $\begin{array}{l}\begin{array}{l}\text { Savane } \\
\text { herbeuse }\end{array} \\
\end{array}$ \\
\hline $\begin{array}{l}\text { Cultures } \\
\text { annuelles }\end{array}$ & 1 & & & & & & & & & \\
\hline $\begin{array}{l}\text { Forêt } \\
\text { claire }\end{array}$ & 23 & 1 & & & & & & & & \\
\hline $\begin{array}{l}\text { Forêt } \\
\text { galerie }\end{array}$ & 7,7 & 9,8 & 1 & & & & & & & \\
\hline $\begin{array}{l}\text { Forêt } \\
\text { sèche }\end{array}$ & 19,2 & 35,5 & 7,5 & 1 & & & & & & \\
\hline Jachère & 41,8 & 47,5 & 11,3 & 0,217 & 1 & & & & & \\
\hline $\begin{array}{l}\text { Plantation } \\
\text { anacarde } \\
\end{array}$ & 0,4 & 18,2 & 17,1 & 10,7 & 37,8 & 1 & & & & \\
\hline $\begin{array}{l}\text { Plantation } \\
\text { Canne } \\
\text { villageoise }\end{array}$ & 9,1 & 0 & 8,3 & 0 & 0,159 & 22,2 & 1 & & & \\
\hline $\begin{array}{l}\text { Savane } \\
\text { arborée }\end{array}$ & 4 & 30,5 & 12 & 11,3 & 51,7 & 37,7 & 0,19 & 1 & & \\
\hline $\begin{array}{l}\text { Savane } \\
\text { arbustive }\end{array}$ & 40,6 & 20,7 & 8,2 & 17,1 & 52,3 & 38,5 & 14,6 & 44,8 & 1 & \\
\hline $\begin{array}{l}\text { Savane } \\
\text { herbeuse }\end{array}$ & 1 & 0 & 1 & 4,9 & 10,2 & 17,4 & 33,3 & 15,8 & 10,8 & 1 \\
\hline
\end{tabular}

Tableau 5 : Liste des espèces comestibles du site de l'étude.

\begin{tabular}{ll}
\hline Espèces & Organes consommés \\
\hline Anacardium occidentalis & Fruits \\
\hline Annona senegalensis & Fruits \\
\hline Blighia welwitschii & Fruits \\
\hline Carica papaya & Fruits \\
\hline Carissa edulis & Fruits \\
\hline Ceiba pentandra & Feuilles \\
\hline Cissu spopulnea & Fruits \\
\hline Citrus limon & Fruits \\
\hline Deinbollia pinnata & Fruits \\
\hline Detarium microcarpum & Fruits \\
\hline Detarium senegalense & Fruits \\
\hline Dialium guineense & Fruits \\
\hline Diospyros mespiliformis & Fruits \\
\hline Ficus sur & Fruits \\
\hline Flacourtia flavescens & Fruits \\
\hline Gardenia ternifolia & Fruits \\
\hline
\end{tabular}




\begin{tabular}{ll}
\hline Imperata cylindrica & Rhizomes \\
\hline Landolphia dulcis & Fruits \\
\hline Landolphia heudelotii & Fruits \\
\hline Mangifera indica & Fruits \\
\hline Margaritaria discoidea & Fruits \\
\hline Moringa oleifera & Fruits \\
\hline Nauclea latifolia & Fruits \\
\hline Parinari curatellifolia & Fruits \\
\hline Parkia biglobosa & Fruits \\
\hline Phoenix reclinata & Fruits, vin de palme \\
\hline Pouteria alnifolia & Fruits \\
\hline Raphia sudanica & Fruits \\
\hline Saba comorensis & Fruits \\
\hline Saccharum officinarum & Tige \\
\hline Tamarindus indica & Fruits \\
\hline Uapaca togoensis & Fruits \\
\hline Uvariaafzelii & Fruits \\
\hline Uvaria tortilis & Fruits \\
\hline Vitellaria paradoxa & Fruits/Huile \\
\hline Vitex doniana & Fruits \\
\hline Vitex madiens & Fruits \\
\hline
\end{tabular}

Tableau 6: Liste des espèces médicinales rencontrées sur le site de l'étude et les pathologies traitées.

\begin{tabular}{lc}
\hline Espèces & Pathologies traitées et organes utilisés \\
\hline Afzelia africana & Migraine, odèmes, épilepsie \\
\hline Ageratum conyzoides & Cicatrisant (Feuilles), Ulcère gastrique (plante entière) \\
\hline Albizia ferruginea & Hémorroïde (Feuilles) \\
\hline Alchornea cordifolia & Ulcère gastrique (écorce) \\
\hline Anacardium occidentalis & Ecorce, Ulcère gastrique \\
\hline Anchomanes difformis & Hémorrö̈de (Tubercule) \\
\hline Antiaris toxicaria & Cicatrisant (écorces) \\
\hline Azadirachta indica & Fatigue, paludisme, ictère \\
\hline Bambusa vulgaris & Fièvre typhoïde, Paludisme, Diabète (Feuille) \\
\hline Bridelia ferruginea & Paxatif (écorce de tige) \\
\hline Cassia sieberiana DC & Paludisme (Racines) \\
\hline Ceiba pentandra & Accès fébrile, toux, maux de cœur (feuilles \&écorce) \\
\hline Chromolaena odorata & Cicatrisant, maux de ventre (feuilles) \\
\hline Ficus exasperata & Extraction d'épine (sève) \\
\hline Khaya anthotheca & Anémie (écorce)/ Ulcère gastrique (Ecorce) \\
\hline Khaya senegalensis & Anémie (écorce) \\
\hline
\end{tabular}




\begin{tabular}{lc}
\hline Lannea nigritana & Mal de ventre (Feuilles et Fruits) \\
\hline Mangifera indica & Paludisme (écorces), Toux (écorces) \\
\hline Margaritaria discoidea & Coupe faim (fruits)/Sommeil chronique/ fatigue générale (feuilles) \\
\hline Morinda lucida & Paludisme (Ecorces, racines) \\
\hline Morinda lucida & Maux d'yeux, entéralgie (feuilles \& écorce) \\
\hline Moringa oleifera & Paludisme, céphalée, Typhoïde (Feuilles) \\
\hline Nauclea latifolia. & Paludisme (écorces des racines) \\
\hline Parinari curatellifolia & Cure-dents (tige) \\
\hline Parkia biglobosa & Tension artérielle (Fruits) \\
\hline Parkia biglobosa & Accès fébrile, rougeole, varicelle (feuille ou écorce de tige) \\
\hline Parquetïna nigrescens & Cicatrisant \\
\hline Paullinia pinnata L. & Hypertension artérielle/ mal de ventre (Ecorces) \\
\hline Phyllanthus amarus & Fièvre typhoïde \\
\hline
\end{tabular}

Tableau 7: Liste des espèces utilisées pour divers usages.

\begin{tabular}{ll}
\hline Espèces & Autres usages \\
\hline Afzelia africana & Bois d'œuvre, poterie (valves de la gousse)/ Fourrage \\
\hline Andropogon tectorum & Construction de l'habitat (chaume) \\
\hline Antiaris toxicaria & Confection d'étoffes avec écorces de la racine/Bois d'œuvre \\
\hline Bombax costatum & Bois d'œuvre \\
\hline Cassia sieberiana & Ornementation arbre d'alignement (horticulture) \\
\hline Ceiba pentandra & Bois d'œuvre (fabrication de pirogue) \\
\hline Combretum paniculatum & Ornementation (horticulture) \\
\hline Cussonia arborea & Menuiserie : mobilier domestique \\
\hline Daniellia olivera & Bois d'œuvre \\
\hline Detarium senegalense & Bois d'œuvre \\
\hline Dialium guineense & Ustensile (Fabrication de pilon) \\
\hline Diospyros mespiliformis & Confection d'objets d'art avec le bois de tige \\
\hline Erythrina senegalensis & Construction d'habitat (tronc)/Confection de palissade; (horticulture) \\
\hline Ficus exasperata & Fourrage (Feuilles) \\
\hline Ficus sur & Nourriture pour bétail (feuilles) \\
\hline Imperata cylindrica & Feuilles servant à la confection des toits de cases et en sparterie \\
\hline Lonchocarpus cyanescens & Feuilles utilisées dans la teinture \\
\hline Milicia excelsa & Bois d'œuvre \\
\hline Milicia regia & Bois d'œuvre \\
\hline Morus mesozygia & Bois d'œuvre \\
\hline Parkia biglobosa & Bois d'œuvre \\
\hline Pterocarpus erinaceus & Fourrage (Feuilles) \\
\hline Raphia sudanica & Artisanat \\
\hline Vitex doniana & Fabrication d'un colorant noir (bois, fruits) \\
\hline Vitex madiensis & Fabrication d'un colorant noir (bois, fruits) \\
\hline
\end{tabular}




\section{DISCUSSION}

\section{Richesse et composition floristiques}

La présente étude montre l'existence de différents types de formations végétales dans la zone d'étude. Cette diversité d'écosystèmes fournit de nombreux services écosystémiques aux populations locales de la région. Cependant, les différents écosystèmes présentent des tailles et des niveaux de diversités spécifiques très différents. En Effet, les formations forestières, présentes dans cette zone de savane sont en nombre réduit. Elles se rencontrent généralement sur des sols latéritiques à caractéristiques pédologiques ne favorisant pas le développement des cultures. De ce fait, comparativement aux formations savanicoles qui présentent de grandes superficies, ces formations forestières sont plus riches et diversifiées. La pauvreté relative des formations savanicoles s'expliquerait par deux faits qui sont étroitement liés. Dans la zone d'étude, ces formations sont très souvent soumises à l'action des feux de brousses, provoquant ainsi de fortes perturbations au niveau de la strate arborescente. Cette affirmation se justifie par cette assertion de Ballouche (2005) qui indique de façon générale que le feu favorise les herbacées, surtout les graminées aux dépens des arbres en ouvrant les formations végétales et en créant donc un espace offrant suffisamment de lumières pour le développement de ces espèces herbacées. La seconde cause de la perte de la diversité des savanes est liée à l'utilisation de ces biotopes pour la mise en place d'exploitations céréalières et de certaines cultures pérennes telles que les plantations d'Anacardium occidentale. Plusieurs auteurs, notamment Hawthorne (1996), SODEFOR (1996), Adou Yao et al. (2002) et Adou Yao et al. (2005) ont prouvé que la transformation des espaces naturels en zones de cultures entraîne une réduction de la composition floristique. Diby et al. (2008) vont dans le même sens en faisant remarquer que la faible richesse des savanes pourrait avoir une origine édaphique ou anthropique. En effet, chaque année, les savanes sont parcourues par des feux qui ont un caractère dommageable sur ces dernières en fonction de la période. Par ailleurs, certains auteurs décrivent les formations savanicoles comme étant la résultante de la dégradation des forêts sèches (Aubreville 1949, 1962; Schnell 1976; Anhuf 1994 ; 1996). En revanche, pour d'autres comme Filleron (2000) en pays Sénoufo ces bois expriment, non une survivance du passé mais plutôt l'expression d'un potentiel. La dominance des microphanérophytes dans la zone d'étude est due à la présence de ces espèces aussi bien dans les formations forestières que savanicoles. Vroh (2013) a démontré à partir de tests de corrélations que l'abondance des microphanérophytes entrainerait une baisse du nombre d'espèces de chaméphytes et de thérophytes. Ces espèces caractéristiques des savanes et des cultures sont des espèces héliophiles qui ont besoin de lumière pour leur développement. Eu égard aux formations forestières inventoriés dans la zone d'étude notamment les forêts secondaire, forêts claires, forêts galerie..., il est donc difficile de retrouver une grande quantité de ces espèces héliophiles à cause de l'ombre fourni par les espèces arborescentes. De même, dans les différents types de savanes rencontrés, l'ombre des arbres nuit à la survie des espèces de graminées. Cette affirmation est en concordance avec les résultats de Sheil et al. (2006) qui montrent que, les espèces herbacées pionnières demandent plus de lumière pour leur germination et leurs plantules ne peuvent survivre à l'ombre.

En ce qui concerne le cortège d'espèces à statut particulier, les biotopes inventoriés y enregistrent une faible richesse. Leur faible présence indique que la végétation est fortement perturbée à l'issue des activités anthropiques (Abrou et al., 2019). Alors, la petite proportion de ces espèces à valeur de conservation indique que les formations végétales de la zone d'étude sont des écosystèmes fragiles qu'il faut nécessairement préserver. Myers et al. (2000) soulignent que les espèces endémiques, rares et menacées d'extinction sont celles pour lesquelles les efforts de conservation doivent être prioritaires. Les faibles valeurs d'indices de 
Shannon témoignent que la végétation présente dans l'ensemble de la zone d'étude se trouve dans un état de perturbation avancée. De ce qui précède, l'on peut retenir que pour une gestion durable des ressources végétales, l'accent devra être mis sur les quelques lambeaux de forêts restantes. Au niveau de la répartition phytogéographique, la dominance des espèces GC-SZ et l'abondance des espèces SZ dans l'ensemble des biotopes inventoriés confirment l'appartenance de notre zone d'étude à une zone de savane soudanienne.

\section{Usages des espèces}

Plusieurs des espèces rencontrées sur le site de l'étude présentent un intérêt particulier pour les populations riveraines car elles sont utilisées dans l'alimentation, le traitement de plusieurs pathologies, l'artisanat, le fourrage. Plusieurs auteurs, notamment Taita (2003), Ganaba et al. (2005) et Zerbo et al. (2011) ont également montré qu'une flore est source de nourriture, de produits sanitaires, de matériels de construction, d'outils domestiques et contribue par ailleurs à diversifier les sources de revenus. Sur la base de l'importance de l'utilisation des plantes médicinales, il ressort de la présente étude que les populations locales du site d'étude, dans leur grande majorité, sont tributaires de la médecine traditionnelle locale. En effet, la guerre a freiné la dynamique du système sanitaire national opérationnel, engendrant une charge additionnelle de morbidité et de mortalité induites, et contribuant à une augmentation de la vulnérabilité des populations, face aux maladies. Ainsi, pour prévenir et/ou guérir toutes sortes de maladies, les populations du Département de Ferkessédougou ont recours aux plantes médicinales, sur recommandation d'un ou plusieurs tradithérapeutes locaux. Ces espèces végétales deviennent alors un patrimoine à documenter comme l'a recommandé l'Organisation Mondiale de la santé (OMS). Des auteurs comme Cunninghan (1993), Dounias et al. (2000), Kasuku et al. (1999), Ngalamulume et al. (1993) et Tshiamuene et al. (1995) ont fait remarquer que l'usage traditionnel des plantes médicinales constitue la base de la médecine curative des populations à revenus modestes. Les résultats de cette étude ont aussi révélé que les biotopes inventoriés abritent également de nombreuses espèces à usage artisanal. En effet, 27 espèces interviennent dans le secteur de l'artisanat ou assimilé : teintures végétales, bois d'artisanat, objets domestiques, cordages ou liens, savons et cosmétiques. AvocèvouAyisso et al. (2009) ont souligné que ces pratiques sont déjà bien signalées dans d'autres pays en Afrique de l'Ouest comme le Bénin. D'autres usages relevés (cordage ou lien) peuvent s'expliquer par la forte présence de lianes dans ces formations. Cela révèle la bonne connaissance et la valorisation des espèces végétales par les populations locales. Les travaux de Ouôba (2006) ont également montré la profonde connaissance et l'utilisation des ressources végétales par les populations locales dans ce domaine de l'artisanat. Les usages liés à certaines espèces de la flore inventoriée du site de Ziémougoula sont nombreux. Ces informations sur les usages ne sauraient être exhaustives. Elles montrent juste la valeur de certaines espèces de la flore pour les communautés locales vivant sur le site et ses environs. Aussi, 24 espèces utilisées comme plantes alimentaires de cueillette ont été recensées. Les parties consommées varient. Elles concernent les feuilles, les fruits, les tiges, les graines et même des racines. Les espèces dont les fruits sont consommés sont les plus nombreuses. Les études réalisées par (Olivier, 2012) en pays Sénoufo signale également l'usage alimentaire des plantes. Pour cet auteur, ce type d'usage relève de la culture traditionnelle et les plantes alimentaires relèvent principalement du domaine des femmes. Celles-ci agrémentent les repas quotidiens en puisant dans les ressources végétales, comme l'exemple des feuilles utilisées dans la préparation de diverses sauces. Par comparaison à des études antérieures menées par Bognounou (1996), Helmfrid (1997) et Olivier et Sanou (2003) en zone de savane ce sont plutôt les fruits qui sont consommés par les populations et cela ne demande pas de préparation culinaire. Un 
grand nombre d'autres espèces ont été reconnues par les populations et d'après les données bibliographiques comme entrant en partie ou en totalité dans des préparations de médicaments traditionnels. Cependant, sur les parties de la plante utilisée, ces personnes ne souhaitaient pas en dire long. Cette perception de la médecine traditionnelle, s'inscrit dans un constat général rapporté par Ouôba et al. (2006), à savoir que la médecine traditionnelle est un domaine relativement fermé où le savoir sur les plantes médicinales se transmet de père en fils.

\section{Conclusion}

La présente étude a permis de faire un bilan du niveau de diversité de la flore et de la végétation des espaces savanicoles dans la circonscription administrative du Tchologo. $\mathrm{Au}$ total 192 espèces végétales ont été recensées dont la plupart sont des issues de la zone de transition forêt-savane. Parmi ces espèces, 20 ont un statut écologique très particulier dont Uvaria tortilis qui est une espèce exclusive de la flore ivoirienne. La présence de ces espèces peut constituer des indicateurs clés pour le suivi environnemental de la zone d'étude.

\section{CONFLIT D'INTERETS}

Les auteurs de cet article déclarent qu'il n'y a aucun conflit d'intérêts.

\section{CONTRIBUTIONS DES AUTEURS}

AC a contribué à la collecte des données, à la conception et à la structuration de l'étude, à la recherche documentaire et à la rédaction de l'article. MO a participé à la correction du manuscrit. EAN a contribué à la conception du manuscrit. JENA participé à la correction du manuscrit.

\section{REMERCIEMENTS}

La présente étude s'inscrit dans le cadre d'un processus de conservation, de protection et de préservation d'une zone de biodiversité dans la société sucrière SUCAF. Le bon déroulement des travaux au sein de de la zone d'étude a été aussi possible grâce à la collaboration des populations locales de Ferkessédougou.

\section{REFERENCES}

Abrou NEJ, Kouamé D, Adou Yao CY. 2019. Diversité floristique des communautés végétales dans l'espace de la Forêt des Marais Tanoé-Ehy (FMTE), Sud-est de la Côte d'Ivoire. International Journal of Biological and Chemical Sciences, 13(6): 2874-2887.

DOI: https://dx.doi.org/10.4314/ijbcs.v13i6.35

Adou-Yao CY, Dengueadhe S, Blom E, N'Guessan KE, Rompaey RV. 2002. Etude de la diversité floristique dans le Sud du Parc National de Tai. Actes du colloque international, Centre Suisse du 27-29 Aout 2001 (2002). Bioterre, Rev. Inter. Sci. de la Vie et de la Terre, $\left(\mathrm{N}^{\circ}\right.$ special) : 49-58.

Adou-Yao CY, Dengueadhe STK, Blom E, N'Guessan KE, Rompaey RV, Wittebolle G, Bongers F. 2005. Diversité floristique et végétation dans le Parc National de Tai, Côte d'Ivoire. Tropenbos-Côte d'Ivoire, Série 5, $57 \mathrm{p}$.

Aké-Assi L. 1998. Impact de l'exploitation forestière et du développement agricole sur la conservation de la biodiversité biologique en Côte d'Ivoire. Le Flamboyant, 46 : 20 - 21.

Aké-Assi L. 2001. Flore de la Côte d'Ivoire 1, Catalogue, Systématique, Biogéographie et Ecologie : Conservatoire et Jardin Botanique. Boisseria 57: Genève (Suisse).

Aké-Assi L. 2002. Flore de la Côte d'Ivoire 2, Catalogue, Systématique, Biogéographie et Ecologie : Conservatoire et Jardin Botanique. Boisseria 58: Genève (Suisse).

Anhuf VD, Wohlfarth-Bottermann M. 1994. Zeitlicher Vegetations-und Klimawandel in Côte d'Ivoire. Editions Franz Steiner Verlag : Deutsch.

Anhuf D. 1996. Recherches de l'Institut de géographie de Mannheim faites à l'étranger francophone. Geographisches Institut der Universität Mannheim, Mannheim. 
APG, IV. 2016. An update of the Angiosperm Phylogeny Group classification for the orders and families of flowering plants: APG IV. A Botanical Journal of the Linnean Society, 181: 120 p.

Aubréeville A. 1947. Les brousses secondaires en Afrique équatoriale. Bois et forêts des Tropiques, 2 : 24-49.

Aubréville A. 1949. Climats, forêts et désertification de l'Afrique tropicale.

Aubréville A. 1962. Savanisation tropicale et glaciations quaternaires. Laboratoire de phanérogamie du Museum national d'histoire naturelle.

Avocèvou-Ayisso C, Sinsin B, Adégbidi A, Dossou G, Van Damme P. 2009. Sustainable use of non-timber forest products: Impact of fruit harvesting on Pentadesma butyracea regeneration and financial analysis of its products trade in Benin. Forest Ecology and Management, 257(9): 1930-1938. DOI: https://doi.org/10.1016/j.foreco.2009.01. 043

Badjaré B, Kokou K, Bigou-lare N, Koumantiga D, Akpakouma A, Adjayi BM, Abbey AG. 2018. Étude ethnobotanique d'espèces ligneuses des savanes sèches au nord-Togo : diversité, usages, importance et vulnérabilité. BASE., 22(3): 152-171. URL: https://popups.uliege.be:443/17804507/index.php?id=16487

Ballouche A, Dolidon H. 2005. Forêts claires et savanes ouest-africaines: dynamiques et évolution de systèmes complexes à l'interface nature-société. In La Forêt: Enjeux Comparés des Formes D'appropriation, de Gestion et D'exploitation dans les Politiques Environnementales et le Contexte d'Urbanisation Généralisée. Acte du Colloque international Poitiers (16-17 octobre 2003) ; 56-70.

Bognounou O. 1996. La diversité biologique végétale au Burkina Faso. Aspects ethnobotaniques. Rapport d'étude pour l'élaboration de la monographie nationale du Burkina Faso sur la Biodiversité, Burkina Faso.
Cunninghan AB. 1993. African medecinal plants setting priorities at the interface between conservation and primary health care. In People and Plants, UNESCO. Working paper of UNESCO: Paris; 1-50.

Davranche A, Taïbi AN. 2015. Les changements d'occupation et d'usage du sol, des processus multidimensionnels complexes qui affectent la biodiversité. In Pour une Géographie de la Conservation, Mathevet R, Godet L (eds). Editions l'Harmattan.

Dibi HN, Adou Yao CY, N'Guessan KE, Koné M, Sagne YC. 2008. Analyse de la diversité floristique du parc national de la Marahoué, Centre-Ouest de la Côte d'Ivoire. Afrique science, 4(3): 552 - 579.

Dounias E, Rodrigue W, Petit C. 2000. Revue de la littérature ethnobotanique pour l'Afrique centrale et l'Afrique de l'ouest. Bulletin du Réseau Africain d'Ethnobotanique $\quad\left(\mathrm{n}^{\circ} 2\right)$, Unesco. Unesco ; 5-117.

Dro B, Soro D, Koné M, Bakayoko A, Kamanzi K. 2013. Évaluation de l'abondance de plantes médicinales utilisées en médecine traditionnelle dans le nord de la Côte d'Ivoire. Journal of Animal \& Plant Sciences, 17(3): 26312646. http://www.m.elewa.org/JAPS

FAO. 2012. Rapport de l'organisation des Nations Unies pour l'alimentation et l'agriculture sur la situation des forêts en 2012. FAO, Rome.

FAO. 2001. La situation mondiale de l'alimentation et de l'agriculture. FAO, Rome, p. 47.

Filleron JC. 2000. La Cuirasse et le Bois Sacré ou Climax Déchu. Presse univ. du Mirial : Toulouse.

Ganaba S, Ouadba JM, Bognounou O. 2005. Exploitation traditionnelle des végétaux spontanés en région Sahélienne du Burkina Faso. VertigO, 6(2). DOI : https://doi.org/10.4000/vertigo.2783

Gueulou N, Ouattara ND, Konan D, Gnahoré E, Missa K, Bakayoko A. 2018. Diversité floristique et structurale de la forêt galerie du Bandama dans la Réserve Scientifique 
de Lamto en Côte d'Ivoire. Afrique Science, 14(4): 439-452.

Hawthorne WD. 1996. Holes and Sums of Parts in Ghanian Forest: Regeneration Scale and Sustainable Use. Proc. Of the Royal Society of Edinburg 104B: Edinburg.

Jongkind CCH, Suter J. 2004. List of Liberian vascular plants. In Advances in Botanical Knowledge of Liberia Supported by the Liberia Forest Re-assessment Project. Fauna and Flora International: Cambridge, UK; 7-9.

Kasuku W, Lula F, Paulus J, Ngiefu N, Kaluila D. 1999. Contribution à l'inventaire des plantes utilisées pour le traitement du paludisme à Kinshasa (RDC). Revue de Médecine et Pharmacopées Africaines, 13: 95-103.

Keay RWJ, Hepper FN. 1954 -1972. Flora of West Tropical Africa (2 $2^{\text {nd }}$ Edn). Crown Agents: London (UK); 209-521.

Kokou K, Adjossou K, Hamberger K. 2005. Les forêts sacrées de l'aire Ouatchi au Sud-Est du Togo et les contraintes actuelles des modes de gestion locales des ressources forestières. VertigO, 6(3): 110.

DOI :

https://doi.org/10.4000/vertigo.2456

Kouamé NMT, Gnahoua GM, Kouassi KE, Traoré D. 2008. Plantes alimentaires spontanées de la région du Fromager (Centre-ouest de la Côte d'Ivoire) : Fore, habitats et organes consommés. Sciences \& Nature, 5(1): 61-70. DOI : https://doi.org/10.4314/scinat.v5i1.42152

Lebrun JP, Stork AL. 1991-1997. Enumération des Plantes à Fleur d'Afrique Tropicale (Vol. 1, 2, and 3). Conservatoire et Jardin botanique de la ville de Genève : Genève.

Mangenot G. 1951. Une formule simple permettant de caractériser les climats de l'Afrique intertropicale dans leurs rapports avec la végétation. Revue Générale de Botanique, 58: 353-372.

Myers N, Mittermeier RA, Mittermeier CG, Da Fonseca GA, Kent J. 2000. Biodiversity hotspots for conservation priorities. Nature, 403(6772): $853 . \quad$ DOI: $10.1038 / 35051132$.
N'Guessan AE, Kassi NJ, Yao NO, Amani HKB, Gouli GZR, Piponiot C, Irie Bi CZ, Hérault B. 2019. Drivers of biomass recovery in a secondary forest ed landscape of West Africa. Forest Ecology and Managment, 433: 325-331.

Ngalamulume T, Paulus JSJ, Kabeya M, Nlandu SL, Kizika K. 1995. Plantes médicinales à usage domestique cultivées dans deux quartiers de Kinshasa. Revue de Médecine et Pharmacopées Africaine, 9(2): 9-14.

N'Klo O. 2000. Situation des ressources génétiques forestières de la Côte d'Ivoire (Zone de Savanes). Atelier sous régional FAO/IPGRI/CIRAF sur la conservation, la gestion, l'utilisation durable et la mise en valeur des ressources génétiques forestières de la zone sahélienne (du 2224 sept. 1998), Ouagadougou.

O.R.S.T.O.M. 1978. Observation sur les premiers stades de la reconstitution de la forêt dense humide (Sud-ouest de la Côted'Ivoire). Cahier O.R.S.T.O.M., 13: 189267.

Olivier JG. 2012. Trends in Global $\mathrm{CO}_{2}$ Emissions: 2012 Report. PBL Netherlands Environmental Assessment Agency : Netherlands.

Olivier M, Sanou L. 2003. Contribution à l'étude des plantes médicinales des jachères de l'ouest du Burkina Faso. Ethnopharmacologia, 30: 47-59. http://indexmedicus.afro.who.int/on

Ouattara D, Kouamé D, Tiébré MS, Cissé A, N'Guessan KE. 2016. Diversité floristique et usages des plantes dans la zone soudanienne du Nordnord-ouest de la Côte d'Ivoire. Journal of Animal \& Plant Sciences, 31(1): 4815-4830. http://www.m.elewa.org/JAPS

Ouôba P, Lykke AM, Boussim J, Guinko S. 2006. La flore médicinale de la forêt classée de Niangoloko (Burkina Faso). Etudes Flor. Vég. Burkina Faso, 10 : 5-16.

Ramade F. 2005. Eléments d'Ecologie: Ecologie Appliquée (6 ${ }^{\mathrm{ème}}$ édn). Editions Dunod : France. 
Raunkiaer C. 1934. The Life Forms of Plants and Statistical Plant Geography; Being The Collected Papers of $C$. Raunkiaer. Clarendron Press: London (UK).

Schnell R. 1976. Introduction de la Phytogéographie des Pays Tropicaux 3 : La Flore et la Végétation de l'Afrique Tropicale. Editions Gauthier-Villars : France.

Schnell R. 1971. Introduction à la Phytogeographie des Pays Tropicaux: les Problèmes Généraux. Editions GauthierVillars : Paris.

Sheil D, Salim A. 2012. Diversity of locally useful tropical forest wild-plants as a function of species richness and informant culture. Biodiversity and Conservation, 21(3): 687-699.

SODEFOR. 1996. Plan d'aménagement de la forêt classée de Bouaflé. SODEFOR, Ministère de l'agriculture et des ressources animales, p. 3-61.

Sorensen T. 1948. A Method of Establishing Groups of Equal Amplitude in Plant Sociology Based on Similarity of Species Content and Its Application to Analyses of the Vegetation on Danish Commons. Biologiske Skrifter : Denmark.

Taïta P. 2003. Use of woody plants by locals in Mare aux Hippopotames Biosphère Reserve in western Burkina Faso. Biodiversity and Conservation, 12: 12051217.

Tankoano B. 2017. Contribution de la télédétection et des Systèmes d'Informations Géographiques à l'évaluation de l'impact des activités humaines sur la couverture végétale : cas du Parc National des Deux Balé (PNDB), à l'Ouest du Burkina Faso. Thèse de Doctorat, Université Nazi Boni, Burkina Faso.

Tiébré MS, Ouattara D, Vroh BTA, Gnagbo A, N Guessan KE. 2016. Diversité floristique et disponibilité des plantes utilitaires en zone soudanienne de la Côte d'Ivoire. Journal of Applied Biosciences,
102: 9699-9707.

DOI :

http://dx.doi.org/10.4314/jab.v102i1.4

Troupin AS. 1966. Photic activation and experimental data concerning colored stimuli. Neurology, 16(3): 269-269.

Tschimuene NJ, Paulus SJ, Kabeya M, Nlandus L, Kizika K. 1995. Plantes médicinales à usage domestique cultivées dans deux quartiers de Kinshasa. Bull. Méd. Trad. Pharm., 9(2): 9-14.

UICN 2018. IUCN Red List of Threatened Species. UICN, Version 2018.1. <www.iucnredlist.org>.

Vroh BTA, Ouattara D, Kpangui KB. 2014. Disponibilité des espèces végétales spontanées à usage traditionnel dans la localité d'Agbaou, Centre ouest de la Côte d'Ivoire. Journal of Applied Biosciences, 76: 6386-6396. DOI: https://doi.org/10.4314/jab.v76i1.11

Vroh BTA. 2013. Évaluation de la dynamique de la végétation dans les zones agricoles d'Azaguié (Sud-est Côte d'Ivoire). Thèse de Doctorat unique, UFR Biosciences, Université Félix Houphouët-Boigny, Abidjan, Côte d'Ivoire, 208 p.

White F, Bamps P. 1983. La Végétation de l'Afrique: Mémoire Accompagnant la Carte de Végétation de l'Afrique UNESCO/AETFAT/UNSO. ORSTOM: UNESCO-Paris.

Yao NO, N'Guessan AE, Égnankou WM, Sangaré M, N'Guessan KE. 2018. Structure et composition de la végétation en zone sub-soudanaise (Département de Dianra, Nord-Ouest de la Côte d'Ivoire): étude descriptive et comparative. Afrique Science, 14(2): 304-315.

Zerbo P, Millogo-Rasolodimby J, NacoulmaOuédraogo O, Van Damme P. 2011. Plantes médicinales et pratiques médicales au Burkina Faso: cas des Ssanan. Bois et Forêts des Tropiques, 307: 47-53. DOI: https://doi.org/10.19182/bft2011.307.a20 481 